\title{
Periarterial stem villous edema is associated with hypercoiled umbilical cord and stem obliterative endarteritis
}

\author{
Jerzy Stanek \\ Division of Pathology and Laboratory Medicine, Cincinnati Children's Hospital Medical Center, Cincinnati, USA \\ Email: jerzy.stanek@cchmc.org
}

Received 19 September 2013; revised 16 October 2013; accepted 23 October 2013

Copyright (C) 2013 Jerzy Stanek. This is an open access article distributed under the Creative Commons Attribution License, which permits unrestricted use, distribution, and reproduction in any medium, provided the original work is properly cited.

\begin{abstract}
To assess clinicopathologic correlations of a novel placental lesion featuring a distinct ring-like periarterial edema of stem villi (PASE). A retrospective case-control statistical comparison (Yates $\chi^{2}$ or analysis of variance) of 30 clinical and 41 placental features of 100 consecutive placentas with PASE and 100 gestational age-matched cases without PASE, extracted from 2403 placentas from high-risk pregnancies signed out by the author since year 2006. The PASE was seen in $4.2 \%$ of placentas, average gestational age 35.9 weeks (range, 24 - 42 weeks). Frequencies of stem obliterative endarteritis and hypercoiled umbilical cord (coiling index $>0.3$ ) were $23 \%$ vs $5 \%$ ( $p=$ $0.005)$, and $20 \%$ vs $9 \%(p=0.04)$ in the study group and comparative group, respectively. There were no statistically significant differences $(p>0.05)$ between the groups in clinical or other placental variables. The PASE may be linked to chronically abnormal blood flow in umbilical cord arteries and their stem branches and may be a histological placental sign of non-obliterative umbilical cord compromise.
\end{abstract}

Keywords: Obliterative Endarteritis; Periarterial Stem Edema; Placenta; Umbilical Cord Coiling

\section{INTRODUCTION}

Edema can affect all three major placental parts: membranes, umbilical cord and chorionic disc (Figure 1). Membrane edema (Figure 1(A)) is usually meconium associated, and umbilical cord edema (Figures 1(B) and (C)) is linked to umbilical vessel thrombosis, polyhydramnios and maternal diabetes mellitus [1]. Villous edema shows more morphologic and etiopathogenetical di- versities. Most commonly, it diffusely affects the terminal and intermediate villi (Figure 1(D)) in the fetal/placental hydrops $[1,2]$. The diagnostic value of so-called "multifocal edema" of intermediate and terminal villi [3] is debatable, at least to the author, as it may representclusters of immature intermediate villi with reticular stroma, containing fluid-filled stromal channels with prominent Hofbauer cells, seen usually in the center of cotyledons [1] (Figure 1(E)). The split between the villous trophoblastic shell and the villous core is the most important histological feature differentiating the truly hydropic villi (Figure 1(D)) from clusters of immature intermediate villi [4] (Figure 1(E)). The author observed the split phenomenon even in originally hydropic but secondarily fibrotic chorionic villi of retained stillbirth. Villous edema also has to be distinguished from the decreased extracellular matrix of chorionic villi seen diffusely in the preuterine pattern of chronic hypoxic placental injury or, focally, in the uterine type of chronic hypoxic placental injury [5].

The edema of stem villi is also histologically variable and etiopathogenetically diverse. It includes the patchy edema of stem villi, so called stem branch edema (Figure 1(F)), of pregnancies complicated by gestational hypertension and newborn admission to neonatal intensive care units. In some cases it is associated with edema of the umbilical cord [6], and the grossly and microscopically distinct massive stem edema/myxoid change of placental mesenchymal dysplasia [7] (Figure 1(G)). In addition, the author has noticed a histologically distinct, ring like adventitial periarterial stem villous edema (PASE) in his placental material (Figures 1(H) and 2(A)). The lesion differs from the stem branch edema (Figure $\mathbf{1 ( F ) ) ~ b y ~ t h e ~ l o c a l i z a t i o n ~ o f ~ t h e ~ e d e m a ~ a r o u n d ~ t h e ~ v i l l o u s ~}$ arteries. So far, the PASE was shown to strongly and positively correlate with gestational age [8], and, by a clus- 


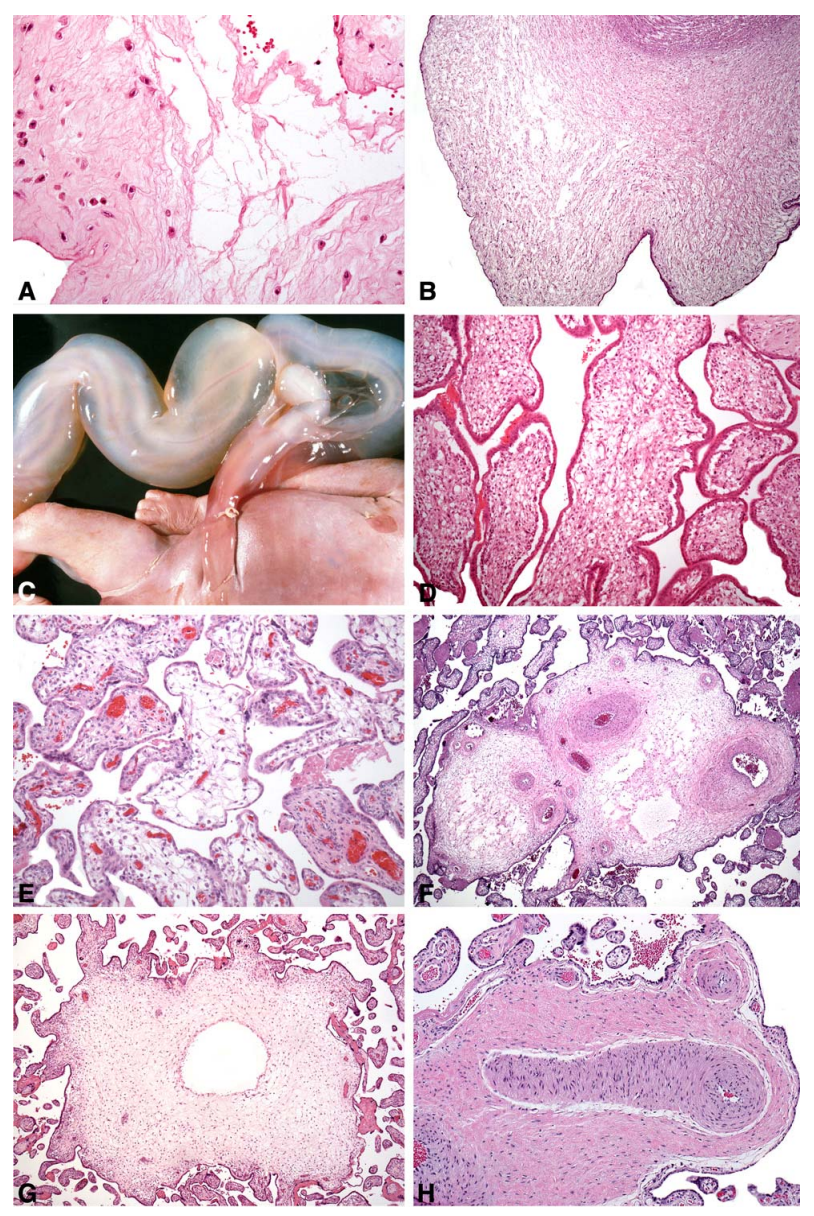

(A) Amnion edema of meconium staining in placental membranes, 41 weeks, cesarean section for fetal distress, $\times 20$; (B) Focal edema of the umbilical cord, 31 weeks, EXIT to airway, polyhydramnios, giant cervical teratoma, $\times 4$; (C) Massive umbilical cord edema, 26 weeks, stillbirth; (D) Hydrops of parvovirus B19 infection, 19 weeks, stillbirth. Edema of intermediate villi is slightly obscured by the stillbirth-related fibrosis, but splits between the trophoblastic shell and the villous core are seen, ×20; (E) Mature and immature intermediate chorionic villi (NOT edematous), 32 weeks pregnancy, EXIT to airway for cervical teratoma, $\times 20$; (F) Stem edema of placental hydrops (stem branch edema, NOT PASE), 33 weeks, EXIT to airway for CHAOS (laryngeal atresia), $\times 4$; (G) Stem edema with cistern formation and myxomatous change of villous stroma of placental mesenchymal dysplasia (NOT PASE), termination of pregnancy, 20 weeks, $\times 4$; $(\mathrm{H})$ Stem perivascular edema (PASE), 39 weeks, large macrocystic lymphatic malformation, cesarean section, $\times 10$.

Figure 1. Placental edema (the objective magnifications given).

tering analysis, to statistically and significantly associate with several clinical and placental factors [9], but has not been analyzed separately yet. Consequently, the retrospective case-control study of the clinicopathologic correlations of the PASE was the aim of this study.

\section{MATERIALS AND METHODS}

The study has been approved by the Institutional Review Board. This is a retrospective case-controlled analysis of cases selected from the database of 2403 placentas, consecutively signed out by the author since year 2006, i.e. the time period during which PASE was consistently recorded in placental reports. The placentas had been submitted for examination because of high risk nature of pregnancy, fetal distress, and poor condition of the neonate, operative delivery, or grossly abnormal placenta, according to the institutional policies. Placental examination was performed according to generally accepted criteria [1,3]. Specifically, the placentas were examined fresh, sliced at $1 \mathrm{~cm}$ intervals, and after the buffered formalin fixation, at least two sections of placental membrane rolls and umbilical cords, and at least two paracentral full thickness chorionic disc sections were routinely taken if no gross lesions were identified. All grossly seen lesions were additionally sampled.

That was followed by routine paraffin embedding, cutting and staining with hematoxylin and eosin. Definitions of clinical conditions [10] and placental diagnoses used in this study were either standard [1,2] or same as in the previous author's publications [5,9]. For the purpose of this analysis, the diagnosis of obliterative endarteritis (OA) was made only if it was seen in at least $50 \%$ of stem villi and obliterated totally or almost totally the vascular lumens.

A cohort of 100 most recently signed placentas with periarterial stem edema involving $>90 \%$ arterial circumference in at least $50 \%$ of stem villi (arbitrary cut-off points), diagnosed as the PASE, formed the study group. The comparative group comprised 100 placentas without the PASE, signed out in same period of time and matched by gestational age but nothing else, to find potential clinicoplacental differences. 30 clinical and 41 placental features were statistically compared between the cases and the comparative group. The Yates chisquare or analysis of variance (ANOVA) was used for statistical analysis, where appropriate and $p<0.05$ was regarded statistically significant.

\section{RESULTS}

The PASE incidence was $4.2 \%$ in the study material. Although the PASE can occasionally be seen in a few stem villi of normal placentas, it showed statistically significant associations only when it was present in more than $50 \%$ stem villi and involved more than $90 \%$ of vessel circumference. Frequencies of 71 clinical and placental variables of our data set are presented in Table 1. The average gestational age was $35.9 \pm 4.2$ weeks (range 24 - 42 weeks) in both the cases and the comparative group, as the cases were gestational age matched. There were no statistically significant differences $(p>0.05)$ between the groups in clinical parameters. Of placental parameters, frequencies of the stem OA and hypercoiled umbilical cord (coiling index $>0.3$ coils/cm) [11] were 23 vs $5 \%(p=0.005)$, and 20 vs $9 \%(p=0.04)$ in the cases and the controls, respectively. 
Table 1. Differences between the cases (periarterial stem edema) and the controls (no stem periarterial edema).

\begin{tabular}{|c|c|c|}
\hline & Cases & Controls \\
\hline Number of cases & 100 & 100 \\
\hline \multicolumn{3}{|l|}{ A. Clinical variables } \\
\hline $\begin{array}{l}\text { Gestational age (weeks, } \\
\text { average } \pm \text { standard deviation) }\end{array}$ & \multicolumn{2}{|c|}{$35.9 \pm 4.2$} \\
\hline Poor or absent prenatal care & 4 & 2 \\
\hline Substance abuse & 12 & 6 \\
\hline Gestational hypertension & 2 & 2 \\
\hline Preeclampsia & 11 & 7 \\
\hline Mild & 6 & 4 \\
\hline Severe & 3 & 3 \\
\hline $\begin{array}{l}\text { HELLP (Hemolysis, Elevated Liver } \\
\text { enzymes, Low Platelets) }\end{array}$ & 2 & 0 \\
\hline Chronic hypertension & 0 & 2 \\
\hline Maternal diabetes mellitus & 12 & 5 \\
\hline Oligohydramnios & 4 & 8 \\
\hline Polyhydramnios & 5 & 2 \\
\hline Premature rupture of membranes & 11 & 11 \\
\hline Antepartum hemorrhage & 19 & 15 \\
\hline Meconium (clinical) & 16 & 14 \\
\hline Thin & 6 & 3 \\
\hline Thick & 10 & 11 \\
\hline Abnormal fetal heart rate tracing ${ }^{\mathrm{a}}$ & 28 & 26 \\
\hline Abnormal umbilical artery Dopplers & 2 & 6 \\
\hline Induction of labor & 17 & 14 \\
\hline Cesarean section & 57 & 45 \\
\hline $\begin{array}{l}\text { One minute Apgar score } \\
\text { (average } \pm \text { standard deviation) }\end{array}$ & $6.1 \pm 3.2$ & $6.3 \pm 3.6$ \\
\hline $\begin{array}{l}\text { Five minutes Apgar score } \\
\text { (average } \pm \text { standard deviation) }\end{array}$ & $7.7 \pm 3.4$ & $7.3 \pm 3.5$ \\
\hline Perinatal mortality & 12 & 10 \\
\hline Neonatal & 8 & 4 \\
\hline Nonmacerated stillbirth & 2 & 1 \\
\hline Macerated stillbirth & 2 & 5 \\
\hline Multiple pregnancy & 9 & 9 \\
\hline $\begin{array}{l}\text { Fetal weight (grams, } \\
\text { average } \pm \text { standard deviation) }\end{array}$ & $2792.5 \pm 827$ & $2574.4 \pm 1044$ \\
\hline Fetal growth restriction ${ }^{\mathrm{b}}$ & 12 & 15 \\
\hline Umbilical cord compromise ${ }^{c}$ & 3 & 7 \\
\hline Congenital malformations & 6 & 2 \\
\hline $\begin{array}{l}\text { Abnormal 3rd stage of labor } \\
\text { (prolonged, hemorrhage) }\end{array}$ & 8 & 4 \\
\hline
\end{tabular}

B. Placental variables

Placental weight (grams, average \pm standard deviation)

$475 \pm 177444 \pm 174$

Inflammation

Acute chorioamnionitis

Maternal inflammatory response

27

$14 \quad 19$

Fetal inflammatory response

$13 \quad 14$

Villitis of unknown etiology

$13 \quad 17$

Plasma cell deciduitis

$4 \quad 7$

Hypoxia-related lesions

Fetal

Erythroblastosis of fetal blood

$12 \quad 5$

Meconium (histological)

Maternal

Villous infarction ( $>5 \%$ of placental parenchyma)

Hypertrophic decidual arteriolopathy

Atherosis of spiral arterioles

Laminar necrosis of membranes ${ }^{\mathrm{d}}$

Patterns of diffuse hypoxic injury

Preuterine

Uterine

Postuterine

Membrane microscopic chorionic pseudocysts

Chorionic disc microscopic

chorionic pseudocysts ${ }^{\mathrm{f}}$

Maternal floor multinucleate trophoblastic giant cells

Excessive amount of extravillous trophoblasts in chorionic disc$$
4
$$$$
4-9
$$$$
14 \quad 14
$$$$
34
$$$$
20 \quad 23
$$$$
19 \quad 13
$$$$
64
$$$$
54
$$$$
8 \quad 5
$$$$
10 \quad 18
$$

Massive perivillous fibrin deposition (>30\% of placental parenchyma)

Chorangiosis

Obliterative endarteritis

Intervillous thrombus

Retroplacental hematoma

Intravillous hemorrhage

Choriodecidual hemosiderosis

Hydrops-related changes

Villous edema

Villous hemosiderosis

Lesions related to decreased/absent fetal blood flow

Luminal vascular abnormalities of chorionic villi 


\begin{tabular}{|c|c|c|}
\hline \multicolumn{3}{|l|}{ Continued } \\
\hline Diffuse villous fibrosis & 4 & 5 \\
\hline Fetal vascular thrombi & 20 & 12 \\
\hline Cluster(s) of at least 3 avascular chorionic villi & 10 & 11 \\
\hline Hemorrhagic endovasculitis & 5 & 8 \\
\hline Intimal cushions in stem/chorionic veins & 9 & 6 \\
\hline \multicolumn{3}{|l|}{ Other } \\
\hline Hypercoiled umbilical cord ${ }^{\mathrm{g}}$ & 20 & 9 \\
\hline Hypocoiled umbilical cord & 9 & 7 \\
\hline Umbilical cord edema & 3 & 3 \\
\hline Other umbilical cord abnormalities ${ }^{\mathrm{h}}$ & 22 & 18 \\
\hline Placenta creta & 7 & 5 \\
\hline Amnion nodosum & 3 & 2 \\
\hline Marginate or vallate placenta & 5 & 4 \\
\hline Gross chorionic cyst(s) & 2 & 0 \\
\hline Succenturiate lobe & 2 & 2 \\
\hline Chorangioma & 1 & 4 \\
\hline \multicolumn{3}{|c|}{$\begin{array}{l}p<0.05 \text { in bold font by Yates } \chi^{2} \text {; }{ }^{\mathrm{a}} \text { Abnormal non stress test and/or abnormal } \\
\text { contraction stress test and/or abnormal intrapartum cardiotocography (pro- } \\
\text { longed bradycardia and/or prolonged tachycardia and or decrease of fetal } \\
\text { heart rate variability and/or late decelerations); ' }{ }^{\mathrm{b}} \text { Birth weight }<10 \text { centile, } \\
\text { c } \text { Variable decelerations, encirclement, true knot, prolapse; }{ }^{\mathrm{d}} \text { At least } 10 \% \text { of } \\
\text { membrane rolls; }{ }^{\mathrm{e}} \text { At least } 3 \text { pseudocysts per membrane roll; }{ }^{\mathrm{f}} \text { At least } 3 \\
\text { pseudocystsper a section of grossly unremarkable chorionic disc; }{ }^{\mathrm{g}} \text { The um- } \\
\text { bilical cord coiling index }>0.3 \text { coils/cm; }{ }^{\mathrm{h}} \text { Too long, too short, too thin, stric- } \\
\text { ture, aneurysm, varix, hematoma, vessel unprotected by Wharton jelly, } \\
\text { chorda, ulcer, barber pole funisitis, amniotic band, meconium toxicity, mar- } \\
\text { ginal insertion, velamentous insertion, furcate insertion. }\end{array}$} \\
\hline
\end{tabular}

\section{DISCUSSION}

To the several types of placental edema, the author added the PASE which histologically features a rarefaction of villous stroma around the stem artery but not the vein (Figure 2(C)) or, less frequently, a distinct and sharply demarcated split between the vessel wall and villous stroma (Figure 1(H)). The current analysis showed the statistically significant association of the PASE with the OA, and umbilical cord hypercoiling (Table 1), but no statistically significant differences between the individual clinical conditions and other placental lesions were found.

The name "OA" is a misnomer because it is not an inflammatory lesion but, at this point, the author chose to keep the name rather than to change it. On hematoxylin and eosin stained slides, it features swelling of endothelial cells with clear cytoplasm, and frequent hobnailing [2], totally (Figures 2(B) and (C)) or partially (Figure 2(D)) obliterating the artery but not the vein lumen (Figure 2(D)). By electron microscopy, the OA features herniation of smooth muscle cytoplasm into the vascular lumen [2] which can also be appreciated by the smooth

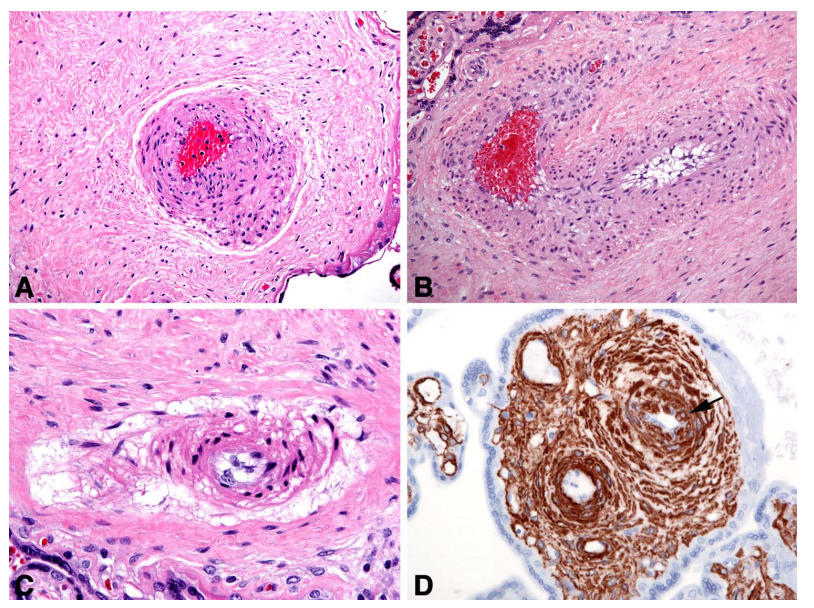

(A) Periarterial edema (PASE) without obliterative endarteritis, 40 weeks, neonate with stroke in the medial cerebral artery distribution, normal umbilical cord coiling, $\times 20$; (B) Obliterative endarteritis without periarterial stem edema, 38 weeks, maternal diabetes mellitus, EXIT-to-airway resection of congenital pulmonary airway malformation, $\times 20$; (C) Obliterative endarteritis and perivascular stem edema (PASE), 35 weeks, twin pregnancy, hypercoiled umbilical cord, $\times 40$; (D) Partial obliterative endarteritis seen in the right stem artery, smooth muscle actin immunohistochemistry, the same case as (B), the arrow indicates SMA-positive cells of the arterial media herniating into the intima, $\times 40$.

Figure 2. Stem periarterial edema (PASE) versus obliterative endarteritis (objective magnifications are given).

muscle actin immunohistochemistry (Figure 2(D)). The (OA) [2] may occur in rare stem villi in otherwise normal placentas and uncomplicated pregnancies not inferquently, but when prominent, it was linked to various pregnancy complications, not only pre-eclampsia and fetal growth restriction [2], but also preterm placentas with babies subsequently developing necrotizing enterocolitis [12], and smokers (although not significantly) [13]. Our previous reports showed that the lesion was 3 times more frequent in placentas with membrane chorionic microscopic pseudocysts than without them, but not with placental chorionic microcysts in general [14], laminar necrosis of membranes [15] or occult placenta accreta [16]. Therefore, it is associated with clinical conditions and outcomes and placental features of in-utero hypoxia. By multidimensional scaling analysis, the PASE and the OA formed a distinct subcluster within a large and statistically significant clinicoplacental cluster of mixed etiology, containing some hypertensive diseases of pregnancy, placental chronic inflammation and extravillous trophoblastic lesions [9]. In this series, the OA was seen in almost a quarter of the cases, significantly more frequently that $5 \%$ in the comparatives (Table 1) but the PASE and the OA were seen mostly independently (Figures 2(A) and (B)).

The other statistically significant correlate of PASE in this material was hypercoiling of the umbilical cord. The latter was reportedly linked to moderate or severe variable fetal heart rate decelerations [11], atypical variable 
decelerations [17], adverse perinatal outcome (low fetal weight, meconium, low Apgar score) [18], stillbirth [19], fetal growth restriction [20], premature deliveries and maternal cocaine use [21]. Of placental parameters, the hypercoiling of umbilical cord was more frequent with vascular thrombosis and ectasia (when combined with other gross umbilical lesions) [22], stasis-induced thrombotic vasculopathy [22,23], placental maturation defect [19], and clusters of multinucleate trophoblastic giant cells in decidua basalis [24], i.e. the clinical conditions and placental features of in-utero hypoxia. The umbilical cord coiling can modulate blood flow through the umbilical cord noticeably and can induce both intimal and adventitial morphological changes of stem arteries [2]. The absence of clinical features of umbilical cord compromise (including variable decelerations) in the current series, most likely indicates the chronic/prolonged nature of umbilical cord hypercoiling rather than an acute cord accident. It seems therefore that the PASE may be caused by hemodynamic abnormalities of the umbilical blood flow rather than by the maternal factors, in particular the decreased uteroplacental blood flow. The decreased uteroplacental blood flow would rather primarily result in formation of the placental hypoxic lesions as the umbilical blood transports oxygen from the placenta to the fetus and therefore the umbilical cord hypoxemia is not the primary cause of hypoxic placental lesions, as opposed to fetal hypoxic lesions, such as erythroblastosis of fetal blood or meconium staining [25].

In conclusion, the association of the PASE with the OA and umbilical cord hypercoiling may be a histological sign of abnormal blood flow in umbilical cord vessels, particularly in the absence of other placental villous hypoxic or thrombotic lesions. This may be an additional placental sign of umbilical cord compromise which is one of major causes of fetal morbidity and mortality, particularly in term and near-term pregnancies. The absence of associated placental hypoxic lesions further supports this conclusion. The umbilical cord compromise associated with the PASE is non-obliterative as it is not associated with the stasis-inducted villous thrombotic vasculopathy. However, the issue should be further studied.

\section{REFERENCES}

[1] Benirschke, K., Kaufmann, P. and Baergen, R.N. (2006) Pathology of the human placenta. Springer, New York.

[2] Fox, H. and Sebire, N.J. (2007) Pathology of the placenta. Saunders, London.

[3] Naeye, R.L. (1992) Disorders of the placenta, fetus, and neonate. Mosby, St. Louis, 174-179.

[4] Boyd, T.K. and Redline, R.W. (2007) Pathology of the placenta. In: Gilbert-Barness, E., Ed., Potter's Pathology of the Fetus, Infant and Child. Mosby, New York.
[5] Stanek, J. (2012) Utility of diagnosing various histological patterns of diffuse chronic hypoxic placental injury. Pediatric and Developmental Pathology, 15, 13-23. http://dx.doi.org/10.2350/11-03-1000-OA.1

[6] Coulter, J.B., Scott, J.M. and Jordan, M.M. (1975) Oedema of the umbilical cord and respiratory distress in the newborn. BJOG: An International Journal of Obstetrics \& Gynaecology, 82, 453-459. http://dx.doi.org/10.1111/j.1471-0528.1975.tb00669.x

[7] Cohen, M.C., Roper, E.C., Sebire, N.J., Stanek, J. and Anumba, D.O. (2005) Placental mesenchymal dysplasia associated with fetal aneuploidy. Prenatal Diagnosis, 25, 187-192. http://dx.doi.org/10.1002/pd.1103

[8] Stanek, J. and Biesiada, J. (2011) Gestational age correlation of clinical conditions and placental lesions. Placenta, 32, A1-A149.

http://dx.doi.org/10.1016/j.placenta.2011.07.004

[9] Stanek, J. and Biesiada, J. (2012) Clustering of maternal/ fetal clinical conditions and outcomes and placental lesions. American Journal of Obstetrics \& Gynecology, 206, 493.e1-8. http://dx.doi.org/10.1016/j.ajog.2012.03.025

[10] Cunningham, F.G., Leveno, K.J., Bloom, S.L., Hauth, J.C., Gilstrap III, L.C. and Wenstrom, K.D. (2005) Williams obstetrics. 32nd Edition, McGraw-Hill, New York.

[11] Strong Jr., T.H., Jarles, D.L., Vega, J.S. and Feldman, D.B. (1994) The umbilical coiling index. American Journal of Obstetrics \& Gynecology, 170, 29-32. http://dx.doi.org/10.1016/S0002-9378(94)70378-7

[12] Dix, L., Roth-Kleiner, M. and Osterheld, M.C. (2010) Placental vascular obstructive lesions: Risk factor for developing necrotizing enterocolitis. Pathology Research International, 2010, 1-7. http://dx.doi.org/10.4061/2010/838917

[13] van der Veen, E., Walker, S. and Fox, H. (1982) Endarteritis obliterans of the fetal stem arteries of the human placenta: An electron microscopic study. Placenta, 3, 181-190. http://dx.doi.org/10.1016/S0143-4004(82)80051-9

[14] Stanek, J. (2011) Placental membrane and placental disc microscopic chorionic cysts share similar clinicopathologic correlations. Pediatric and Developmental Pathology, 14, 1-9. http://dx.doi.org/10.2350/10-02-0795-OA.1

[15] Stanek, J. (2009) Acute and chronic placental membrane hypoxic lesions. Virchows Archiv, 455, 315-322. http://dx.doi.org/10.1007/s00428-009-0841-8

[16] Stanek, J. and Drummond, Z. (2007) Occult placenta accreta: The missing link in the diagnosis of abnormal placentation. Pediatric and Developmental Pathology, 10, 266-262. http://dx.doi.org/10.2350/06-10-0174.1

[17] Hasagawa, J., Matsuoka, R., Ischizuka, K., et al. (2009) Atypical variable deceleration in the first stage of labor is a characteristic fetal heart-rate pattern for velamentous cord insertion and hypercoiled cord. Journal of Obstetrics and Gynaecology Research, 35, 35-39. http://dx.doi.org/10.1111/j.1447-0756.2008.00863.x

[18] Kashanian, M., Akbarian, A. and Kouhpayehzadeh, J. (2006) The umbilical coiling index and adverse perinatal outcome. International Journal of Gynecology \& Obstet- 
rics, 95, 8-13.

http://dx.doi.org/10.1016/j.ijgo.2006.05.029

[19] de Laat, M.W.M., van der Meu, J.J.C., Visser, G.H.A., Franx, A. and Nikkels, P.G.J. (2007) Hypercoiling of the umbilical cord and placental maturation defect: Associated pathology? Pediatric and Developmental Pathology, 10, 293-299. http://dx.doi.org/10.2350/06-01-0015.1

[20] Nishio, J., Nakai, Y., Mine, M., Imanaka, M. and Ogita, S. (1999) Characteristics of blood flow in intrauterine growthrestricted fetuses with hypercoiled cord. Ultrasound in Obstetrics \& Gynecology, 13, 171-175. http://dx.doi.org/10.1046/j.1469-0705.1999.13030171.x

[21] Rana, J., Ebert, G.A. and Kappy, K.A. (1995) Adverse perinatal outcome in patients with an abnormal umbilical coiling index. Obstetrics \& Gynecology, 85, 573-577. http://dx.doi.org/10.1016/0029-7844(94)00435-G

[22] Parast, M.M., Crum, C.P. and Boyd, T.K. (2008) Placen- tal histologic criteria for umbilical blood flow restriction in unexpected stillbirth. Human Pathology, 39, 948-953. http://dx.doi.org/10.1016/j.humpath.2007.10.032

[23] Redline, R.W. (2004) Clinical and pathological umbilical; cord abnormalities in fetal thrombotic vasculopathy. $\mathrm{Hu}$ man Pathology, 35, 1494-1498. http://dx.doi.org/10.1016/j.humpath.2004.08.003

[24] Stanek, J. and Biesiada, J. (2012) Sensitivity and specificity of finding of multinucleate trophoblastic giant cells in decidua in placentas from high-risk pregnancies. $\mathrm{Hu}$ man Pathology, 43, 261-268. http://dx.doi.org/10.1016/j.humpath.2011.03.012

[25] Stanek, J. (2013) Hypoxic patterns of placental injury. A review. Archives of Pathology \& Laboratory Medicine, 137, 706-720.

http://dx.doi.org/10.5858/arpa.2011-0645-RA 